\title{
Pseudo-Hermitian Quantum Dynamics of Tachyonic Spin-1/2 Particles
}

\author{
U. D. Jentschura and B. J. Wundt \\ Department of Physics, Missouri University of Science and Technology, Rolla, Missouri 65409-0640, USA
}

\begin{abstract}
We investigate the spinor solutions, the spectrum and the symmetry properties of a matrixvalued wave equation whose plane-wave solutions satisfy the superluminal (tachyonic) dispersion relation $E^{2}=\vec{p}^{2}-m^{2}$, where $E$ is the energy, $\vec{p}$ is the spatial momentum, and $m$ is the mass of the particle. The equation reads $\left(\mathrm{i} \gamma^{\mu} \partial_{\mu}-\gamma^{5} m\right) \psi=0$, where $\gamma^{5}$ is the fifth current. The tachyonic equation is shown to be $\mathcal{C P}$ invariant, and $\mathcal{T}$ invariant. The tachyonic Hamiltonian $H_{5}=\vec{\alpha} \cdot \vec{p}+\beta \gamma^{5} m$ breaks parity and is non-Hermitian but fulfills the pseudo-Hermitian property $H_{5}(\vec{r})=P H_{5}^{+}(-\vec{r}) P^{-1}=\mathcal{P} H_{5}^{+}(\vec{r}) \mathcal{P}^{-1}$, where $P$ is the parity matrix and $\mathcal{P}$ is the full parity transformation. The energy eigenvalues and eigenvectors describe a continuous spectrum of planewave solutions (which correspond to real eigenvalues for $|\vec{p}| \geq m$ ) and evanescent waves, which constitute resonances and antiresonances with complex-conjugate pairs of resonance eigenvalues (for $|\vec{p}|<m$ ). In view of additional algebraic properties of the Hamiltonian which supplement the pseudo-Hermiticity, the existence of a resonance energy eigenvalues $E$ implies that $E^{*},-E$, and $-E^{*}$ also constitute resonance energies of $H_{5}$.
\end{abstract}

Accepted for publication by J. Phys. A: Math. Theor. for the special issue on "Quantum Physics with non-Hermitian Operators"

PACS numbers: 95.85.Ry, 11.10.-z, 03.70.+k

\section{INTRODUCTION}

Extensions of the Dirac equation have been discussed in great numbers in the scientific literature, including fieldtheoretical formulations [1]. Notably, there have been efforts to incorporate space-time curvature into the equation [2], and, to enhance the equation for spin-1 particles such as the photon, and to map the Maxwell equations onto Diractype equations [3]. Here, we follow a different route and analyze whether it is meaningful and consistent to modify the mass term in the free Dirac equation so that the energy-momentum dispersion relation changes from $E^{2}=\vec{p}^{2}+m^{2}$ to $E^{2}=\vec{p}^{2}-m^{2}$. The latter dispersion relation would be relevant for superluminal particles, which travel faster than the speed of light. The tachyonic spin- $1 / 2$ equation reads

$$
\left(\mathrm{i} \gamma^{\mu} \partial_{\mu}-\gamma^{5} m\right) \psi(x)=0,
$$

where the $\gamma^{\mu}$ are the Dirac matrices, $\partial_{\mu}=\partial / \partial x^{\mu}$ is the derivative with respect to space-time coordinates, and $\gamma^{5}=\mathrm{i} \gamma^{0} \gamma^{1} \gamma^{2} \gamma^{3}$ is the fifth current [here, $x=(t, \vec{r})$ denotes a space-time four vector]. Because of the occurrence of the Dirac matrices, we propose the name 'tachyonic Dirac equation'. The corresponding Lagrangian density reads

$$
\mathcal{L}=\frac{\mathrm{i}}{2} \bar{\psi} \gamma^{5} \overleftrightarrow{\not} \psi-m \bar{\psi} \psi=\frac{\mathrm{i}}{2}\left(\bar{\psi} \gamma^{5} \gamma^{\mu}\left(\partial_{\mu} \psi\right)-\left(\partial_{\mu} \bar{\psi}\right) \gamma^{5} \gamma^{\mu} \psi\right)-m \bar{\psi} \psi
$$

where $\bar{\psi}=\psi^{+} \gamma^{0}$ is the Dirac adjoint. It is perhaps not immediately obvious that the $\gamma^{5}$ matrix has to be placed with the current matrix $\gamma^{\mu}$ in the Lagrangian; otherwise an inconsistent equation is obtained for $\bar{\psi}$ upon variation with respect to $\psi$.

Indeed, the tachyonic Dirac equation has been indicated by Chodos, Hauser and Kostelecky in Refs. [4] in the equivalent form $\left(\mathrm{i} \gamma^{5} \gamma^{\mu} \partial_{\mu}-m\right) \psi=0$, where the current is modified instead of the mass term (see also Refs. [5, 6]). More recently, the noncovariant (Hamiltonian) form of the equation has been indicated in Refs. [7,8], with a preliminary ansatz for its solution. Very recently, the equation has been used in the context of the Gross-Neveu model in Ref. [9]. However, neither the complete set of solutions, nor the spectrum, nor the symmetries of the equation have been fully investigated in the literature to the best of our knowledge.

An important observation made in the current work concerns the Hamiltonian

$$
H_{5}=\vec{\alpha} \cdot \vec{p}+\beta \gamma^{5} m
$$

which is obtained from the tachyonic Dirac equation. Here, $\vec{\alpha}=\gamma^{0} \vec{\gamma}$ and $\beta=\gamma^{0}$, with the identification of the Dirac matrices discussed below in Eq. (6). The Hamiltonian $H_{5}$ breaks parity and is non-Hermitian but fulfills the pseudo-Hermitian equation

$$
H_{5}(\vec{r})=P H_{5}^{+}(-\vec{r}) P^{-1}=\mathcal{P} H_{5}^{+}(\vec{r}) \mathcal{P}^{-1}
$$


where $P$ is the parity matrix and $\mathcal{P}$ is the full parity transformation. While the scalar product induced by $\mathcal{P}$ is not positive semi-definite, a few essential properties of the Hamiltonian persist under the pseudo-Hermiticity and help to characterize the spectrum. We thus extend recent work on non-Hermitian, but pseudo-Hermitian or $\mathcal{P} \mathcal{T}$-symmetric Hamiltonians [10-20] which have also been applied in the field-theoretical context [21-23].

We proceed as follows: In Sec. II, we analyze the transformation properties of the covariant formulation of the tachyonic Dirac equation under charge conjugation, parity transformation and time reversal. The propagator and the explicit solutions of the tachyonic Dirac equation are analyzed in Sec. III. Finally, conclusions are drawn in Sec. IV. Units with $\hbar=c=\epsilon_{0}=1$ are used throughout the paper.

\section{DISCRETE SYMMETRIES: $\mathcal{C}, \mathcal{P}$, AND $\mathcal{T}$}

\section{A. Charge Conjugation}

We start from

$$
\left[\gamma^{\mu}\left(\mathrm{i} \partial_{\mu}-e A_{\mu}\right)-\gamma^{5} m\right] \psi=0
$$

where the $\gamma^{\mu}$ are the Dirac $\gamma$ matrices, which we use in the Dirac representation,

$$
\gamma^{0}=\left(\begin{array}{cc}
\mathbb{1}_{2 \times 2} & 0 \\
0 & -\mathbb{1}_{2 \times 2}
\end{array}\right), \quad \vec{\gamma}=\left(\begin{array}{cc}
0 & \vec{\sigma} \\
-\vec{\sigma} & 0
\end{array}\right), \quad \gamma^{5}=\left(\begin{array}{cc}
0 & \mathbb{1}_{2 \times 2} \\
\mathbb{1}_{2 \times 2} & 0
\end{array}\right) \text {. }
$$

The Dirac $\gamma^{\mu}$ matrices fulfill the following anticommutator relations,

$$
\left\{\gamma^{\mu}, \gamma^{\nu}\right\}=2 g^{\mu \nu}
$$

where $g^{\mu \nu}=\operatorname{diag}(1,-1,-1,-1)$ is the space-time metric. Transposition and complex conjugation leaves $\gamma^{5}$ invariant,

$$
\psi^{+}\left(\left(\gamma^{\mu}\right)^{+}\left(-\mathrm{i} \overleftarrow{\partial}_{\mu}-e A_{\mu}\right)-\gamma^{5} m\right)=0
$$

where the partial derivative acts on the left. The Dirac adjoint is introduced as

$$
\left(\psi^{+} \gamma^{0}\right) \gamma^{0}\left(\left(\gamma^{\mu}\right)^{+}\left(-\mathrm{i} \overleftarrow{\partial}_{\mu}-e A_{\mu}\right)-\gamma^{5} m\right) \gamma^{0}=0
$$

Using the identities $\gamma^{0}\left(\gamma^{\mu}\right)^{+} \gamma^{0}=\gamma^{\mu}$ and $\gamma^{0} \gamma^{5} \gamma^{0}=-\gamma^{5}$, one writes

$$
\bar{\psi}\left(\gamma^{\mu}\left(-\mathrm{i} \overleftarrow{\partial}_{\mu}-e A_{\mu}\right)+\gamma^{5} m\right)=0
$$

A further transposition leads to

$$
\left(\left(\gamma^{\mu}\right)^{\mathrm{T}}\left(-\mathrm{i} \partial_{\mu}-e A_{\mu}\right)+\gamma^{5} m\right) \bar{\psi}^{\mathrm{T}}=0 .
$$

Introducing the charge conjugation matrix $C$ with the property

$$
C\left(\gamma^{\mu}\right)^{\mathrm{T}} C^{-1}=-\gamma^{\mu}
$$

the charge conjugation of the fifth current is calculated symbolically as

$$
\begin{aligned}
& C\left(\gamma^{5}\right)^{\mathrm{T}} C^{-1}=C \mathrm{i}\left(\gamma^{3}\right)^{\mathrm{T}}\left(\gamma^{2}\right)^{\mathrm{T}}\left(\gamma^{1}\right)^{\mathrm{T}}\left(\gamma^{0}\right)^{\mathrm{T}} C^{-1} \\
& =\mathrm{i} C\left(\gamma^{3}\right)^{\mathrm{T}} C^{-1} C\left(\gamma^{2}\right)^{\mathrm{T}} C^{-1} C\left(\gamma^{1}\right)^{\mathrm{T}} C^{-1} C\left(\gamma^{0}\right)^{\mathrm{T}} C^{-1} \\
& =\mathrm{i}\left(-\gamma^{3}\right)\left(-\gamma^{2}\right)\left(-\gamma^{1}\right)\left(-\gamma^{0}\right) \\
& =\mathrm{i} \gamma^{3} \gamma^{2} \gamma^{1} \gamma^{0}=\mathrm{i} \gamma^{0} \gamma^{1} \gamma^{2} \gamma^{3}=\gamma^{5}
\end{aligned}
$$

Therefore, $\psi^{\mathcal{C}}=C \bar{\psi}^{\mathrm{T}}$ fulfills the charge conjugate tachyonic Dirac equation,

$$
\left[\gamma^{\mu}\left(\mathrm{i} \partial_{\mu}+e A_{\mu}\right)+\gamma^{5} m\right] \psi^{\mathcal{C}}=0 \text {. }
$$

As a result of the calculation of the Dirac adjoint $\left(\gamma^{5} \rightarrow \gamma^{0} \gamma^{5} \gamma^{0}=-\gamma^{5}\right)$ and the charge conjugation using the $C$ matrix, the term with the $\gamma^{5}$ matrix has changed sign. In contrast to the ordinary Dirac equation [24], the tachyonic equation is not $\mathcal{C}$ invariant. 


\section{B. Parity}

Again, we start from

$$
\left(\mathrm{i} \gamma^{\mu} \partial_{\mu}-\gamma^{5} m\right) \psi(x)=\left(\mathrm{i} \gamma^{0} \partial_{0}+\mathrm{i} \gamma^{i} \partial_{i}-\gamma^{5} m\right) \psi(x)=0
$$

Under parity, $x \rightarrow x_{P}=(t,-\vec{r})$ and so

$$
\left(\mathrm{i} \gamma^{0} \partial_{0}-\mathrm{i} \gamma^{i} \partial_{i}-\gamma^{5} m\right) \psi\left(x_{P}\right)=0
$$

One introduces a parity transformation matrix $P$, where $P=\gamma^{0}$ in the Dirac representation, with the property

$$
P \gamma^{0} P^{-1}=\gamma^{0}, \quad P \gamma^{i} P^{-1}=-\gamma^{i}, \quad P \gamma^{5} P^{-1}=-\gamma^{5},
$$

where again $i=1,2,3$ is spatial. So, $\psi^{\mathcal{P}}(x)=P \psi\left(x_{P}\right)$ fulfills the parity transformed tachyonic Dirac equation,

$$
P\left(\mathrm{i} \gamma^{0} \partial_{0}-\mathrm{i} \gamma^{i} \partial_{i}-\gamma^{5} m\right) P^{-1} \psi^{\mathcal{P}}(x)=0
$$

which can be rewritten as

$$
\left(\mathrm{i} \gamma^{0} \partial_{0}+\mathrm{i} \gamma^{i} \partial_{i}+\gamma^{5} m\right) \psi^{\mathcal{P}}(x)=0
$$

In covariant notation, we have

$$
\left(\mathrm{i} \gamma^{\mu} \partial_{\mu}+\gamma^{5} m\right) \psi^{\mathcal{P}}(x)=0
$$

The tachyonic Dirac equation is not parity invariant.

\section{Time Reversal}

Starting from the tachyonic Dirac equation,

$$
\left(\mathrm{i} \gamma^{\mu} \partial_{\mu}-\gamma^{5} m\right) \psi=0
$$

transposition and complex conjugation leads to

$$
\psi^{+}\left(-\mathrm{i}\left(\gamma^{\mu}\right)+\overleftarrow{\partial}_{\mu}-\gamma^{5} m\right)=0
$$

where again the differential operator $\overleftarrow{\partial}_{\mu}$ acts to the left. The insertion of the $\gamma^{0}$ matrix ensures Lorentz covariance and leads to

$$
\left(\psi^{+} \gamma^{0}\right) \gamma^{0}\left(-\mathrm{i}\left(\gamma^{\mu}\right)+\overleftarrow{\partial}_{\mu}-\gamma^{5} m\right) \gamma^{0}=0
$$

which is equivalent to

$$
\bar{\psi}\left(-\mathrm{i} \gamma^{\mu} \overleftarrow{\partial}_{\mu}+\gamma^{5} m\right)=0
$$

We here use the fact that a proper formulation of time reversal must respect the fact that the in and out states interchange under $\mathcal{T}$. We thus have to formulate the time reversal in terms of the adjoint of the Dirac equation (see Sec. 4.4.2 of Ref. [25]). Again, we transpose and write

$$
\left(-\mathrm{i}\left(\gamma^{\mu}\right)^{\mathrm{T}} \partial_{\mu}+\gamma^{5} m\right) \bar{\psi}^{\mathrm{T}}=0
$$

We introduce the time reversal matrix $T$, with the properties $(\mathcal{T}$ means time reversal, $T$ is the time reversal matrix, and $\mathrm{T}$ denotes the transpose),

$$
T\left(\gamma^{0}\right)^{\mathrm{T}} T^{-1}=\gamma^{0}, \quad T\left(\gamma^{i}\right)^{\mathrm{T}} T^{-1}=-\gamma^{i}
$$


The time reversal of the fifth current is calculated as

$$
\begin{aligned}
& T\left(\gamma^{5}\right)^{\mathrm{T}} T^{-1}=T \mathrm{i}\left(\gamma^{3}\right)^{\mathrm{T}}\left(\gamma^{2}\right)^{\mathrm{T}}\left(\gamma^{1}\right)^{\mathrm{T}}\left(\gamma^{0}\right)^{\mathrm{T}} T^{-1} \\
& =\mathrm{i} T\left(\gamma^{3}\right)^{\mathrm{T}} T^{-1} T\left(\gamma^{2}\right)^{\mathrm{T}} T^{-1} T\left(\gamma^{1}\right)^{\mathrm{T}} T^{-1} T\left(\gamma^{0}\right)^{\mathrm{T}} T^{-1} \\
& =\mathrm{i}\left(-\gamma^{3}\right)\left(-\gamma^{2}\right)\left(-\gamma^{1}\right)\left(+\gamma^{0}\right) \\
& =-\mathrm{i} \gamma^{3} \gamma^{2} \gamma^{1} \gamma^{0}=-\mathrm{i} \gamma^{0} \gamma^{1} \gamma^{2} \gamma^{3}=-\gamma^{5} .
\end{aligned}
$$

The time-reversed tachyonic Dirac equation thus reads as

$$
\left(\mathrm{i} \gamma^{\mu} \partial_{\mu}-\gamma^{5} m\right) \psi^{\mathcal{T}}(x)=0
$$

where $\psi^{\mathcal{T}}(x)=T \bar{\psi}^{\mathrm{T}}\left(x_{T}\right)$ with $x=(-t, \vec{r})$. As a result of the replacements $\gamma^{5} \rightarrow \gamma^{0} \gamma^{5} \gamma^{0}$ and $\gamma^{5} \rightarrow T \gamma^{5} T-1$, the tachyonic Dirac equation is seen to be $\mathcal{T}$ invariant.

\section{Overview of the Symmetry Properties}

A brief summary is in order. The charge conjugation matrix fulfills [24, 26]

$$
C\left(\gamma^{\mu}\right)^{\mathrm{T}} C^{-1}=-\gamma^{\mu}
$$

In the Dirac representation, a possible choice is $C=\mathrm{i} \gamma^{2} \gamma^{0}$. The parity transformation fulfills

$$
P \gamma^{0} P^{-1}=\gamma^{0}, \quad P \gamma^{i} P^{-1}=-\gamma^{i} .
$$

In the Dirac representation, we may choose $P=\gamma^{0}$. The time reversal operation fulfills

$$
T\left(\gamma^{0}\right)^{\mathrm{T}} T^{-1}=\gamma^{0}, \quad T\left(\gamma^{i}\right)^{\mathrm{T}} T^{-1}=-\gamma^{i}
$$

In the Dirac representation of the $\gamma$ matrices, one can choose $T=\mathrm{i} \gamma^{2} \gamma^{5}$. The matrices $C, P$ and $T$ fulfill the relations $C^{\mathrm{T}}=C^{-1}, P^{\mathrm{T}}=P^{-1}, T^{\mathrm{T}}=T^{-1}$, where by $\mathrm{T}$ we denote the transpose. A representation of $\mathcal{C P} \mathcal{T}$ is given by

$$
C P T=\gamma^{5}
$$

This implies that

$$
(C P T) \gamma^{\mu}(C P T)^{-1}=-\gamma^{\mu}
$$

a relation which has been given on page 239 of the textbook [27] and in Eq. (40.48) of Ref. [28]. We also recall the transformation properties of $\gamma^{5}$ just derived,

$$
\begin{array}{r}
C\left(\gamma^{5}\right)^{\mathrm{T}} C^{-1}=\gamma^{5}, \\
P \gamma^{5} P^{-1}=-\gamma^{5}, \\
T\left(\gamma^{5}\right)^{\mathrm{T}} T^{-1}=-\gamma^{5} .
\end{array}
$$

We note that the transformation property for $T$ depends on our precise formulation of the time reversal operation as detailed in Sec II C. According to Eqs. (14), (20), and (28), the tachyonic Dirac equation is separately $\mathcal{C P}$ invariant, and $\mathcal{T}$ invariant.

\section{SOLUTIONS OF THE TACHYONIC EQUATION}

\section{A. Plane-Wave Solutions and Chirality}

We start from the eigenfunctions of the operator $\vec{\sigma} \cdot \vec{k}$, which are given by

$$
a_{+}(\vec{k})=\left(\begin{array}{c}
\cos \left(\frac{\theta}{2}\right) \\
\sin \left(\frac{\theta}{2}\right) \mathrm{e}^{\mathrm{i} \varphi}
\end{array}\right), \quad a_{-}(\vec{k})=\left(\begin{array}{c}
-\sin \left(\frac{\theta}{2}\right) \mathrm{e}^{-\mathrm{i} \varphi} \\
\cos \left(\frac{\theta}{2}\right)
\end{array}\right)
$$


and fulfill

$$
\begin{gathered}
\frac{\vec{\sigma} \cdot \vec{k}}{|\vec{k}|} a_{ \pm}(\vec{k})= \pm a_{ \pm}(\vec{k}) \\
\sum_{ \pm} a_{ \pm}(\vec{k}) \otimes a_{ \pm}^{+}(\vec{k})=\mathbb{1}_{2}, \quad \sum_{ \pm}(-1)^{ \pm} a_{ \pm}(\vec{k}) \otimes a_{ \pm}^{+}(\vec{k})=\frac{\vec{\sigma} \cdot \vec{k}}{|\vec{k}|},
\end{gathered}
$$

where $\theta$ and $\varphi$ are the polar and azimuthal angles of the wave vector $\vec{k}$. The normalized positive-energy chirality and helicity eigenstates of the massless Dirac equation are

$$
u_{+}(\vec{k})=\frac{1}{\sqrt{2}}\left(\begin{array}{c}
a_{+}(\vec{k}) \\
a_{+}(\vec{k})
\end{array}\right), \quad u_{-}(\vec{k})=\frac{1}{\sqrt{2}}\left(\begin{array}{c}
a_{-}(\vec{k}) \\
-a_{-}(\vec{k})
\end{array}\right) .
$$

These eigenstates immediately lead to plane-wave solutions of the massless Dirac equation, which are also eigenstates of the chirality $\gamma^{5}$ and of the helicity $\vec{\Sigma} \cdot \vec{p} /|\vec{p}|$, where $\vec{\Sigma}$ is the vector of $(4 \times 4)$-spin matrices,

$$
\begin{aligned}
\psi(x) & =u_{ \pm}(\vec{k}) \mathrm{e}^{-\mathrm{i} k \cdot x}, \quad k=(E, \vec{k}), \quad E=|\vec{k}|, \\
\mathrm{i} \gamma^{\mu} \partial_{\mu} \psi(x) & =\gamma^{\mu} k_{\mu} \psi(x)=0, \quad \mathrm{i} \partial_{0} \psi(x)=E \psi(x), \\
\frac{\vec{\Sigma} \cdot \vec{k}}{|\vec{k}|} u_{ \pm}(\vec{k}) & =\gamma^{5} u_{ \pm}(\vec{k})= \pm u_{ \pm}(\vec{k}), \quad \frac{\vec{\Sigma} \cdot \vec{p}}{|\vec{p}|} u_{ \pm}(\vec{k}) \mathrm{e}^{\mathrm{i} \vec{k} \cdot \vec{r}}= \pm u_{ \pm}(\vec{k}) \mathrm{e}^{\mathrm{i} \vec{k} \cdot \vec{r}} .
\end{aligned}
$$

For the negative-energy solutions of the massless Dirac equation, we need

$$
v_{+}(\vec{k})=\frac{1}{\sqrt{2}}\left(\begin{array}{c}
-a_{+}(\vec{k}) \\
-a_{+}(\vec{k})
\end{array}\right), \quad v_{-}(\vec{k})=\frac{1}{\sqrt{2}}\left(\begin{array}{c}
-a_{-}(\vec{k}) \\
a_{-}(\vec{k})
\end{array}\right) .
$$

The negative-energy states fulfill the relations

$$
\begin{aligned}
\phi(x) & =v_{ \pm}(\vec{k}) \mathrm{e}^{\mathrm{i} k \cdot x}, \quad k=(E, \vec{k}), \quad E=|\vec{k}|, \\
\mathrm{i} \gamma^{\mu} \partial_{\mu} \phi(x) & =-\gamma^{\mu} k_{\mu} \phi(x)=0, \quad \mathrm{i} \partial_{0} \phi(x)=-E \phi(x), \\
\frac{\vec{\Sigma} \cdot \vec{k}}{|\vec{k}|} v_{ \pm}(\vec{k}) & =\gamma^{5} v_{ \pm}(\vec{k})= \pm v_{ \pm}(\vec{k}), \quad \frac{\vec{\Sigma} \cdot \vec{p}}{|\vec{p}|} v_{ \pm}(\vec{k}) \mathrm{e}^{-\mathrm{i} \vec{k} \cdot \vec{r}}=\mp v_{ \pm}(\vec{k}) \mathrm{e}^{-\mathrm{i} \vec{k} \cdot \vec{r}} .
\end{aligned}
$$

The subscript of the $v$ vectors is chosen according to the corresponding eigenvalue of the chirality $\gamma^{5}$, i.e., $\gamma^{5} u_{ \pm}= \pm u_{ \pm}$, and $\gamma^{5} v_{ \pm}= \pm v_{ \pm}$. For negative-energy solutions, chirality and helicity are opposite [24]. It is worth noting that the set of four states,

$$
u_{ \pm}(\vec{k}) \mathrm{e}^{\mathrm{i} \vec{k} \cdot \vec{r}}, \quad v_{ \pm}(-\vec{k}) \mathrm{e}^{\mathrm{i} \vec{k} \cdot \vec{r}}
$$

form a complete and orthonormal set of eigenstates of the massless Hamiltonian $H_{0}$, which is a Hermitian operator,

$$
\begin{gathered}
H_{0}=\vec{\alpha} \cdot \vec{p}, \quad \vec{\alpha}=\gamma^{0} \vec{\gamma}, \\
H_{0} u_{ \pm}(\vec{k}) \mathrm{e}^{\mathrm{i} \vec{k} \cdot \vec{r}}=|\vec{k}| u_{ \pm}(\vec{k}) \mathrm{e}^{\mathrm{i} \vec{k} \cdot \vec{r}}, \quad H_{0} v_{ \pm}(-\vec{k}) \mathrm{e}^{\mathrm{i} \vec{k} \cdot \vec{r}}=-|\vec{k}| v_{ \pm}(-\vec{k}) \mathrm{e}^{\mathrm{i} \vec{k} \cdot \vec{r}} \\
u_{\alpha}^{+}(\vec{k}) u_{\beta}(\vec{k})=\delta_{\alpha \beta}, \quad v_{\alpha}^{+}(-\vec{k}) v_{\beta}(-\vec{k})=\delta_{\alpha \beta}, \quad u_{\alpha}^{+}(\vec{k}) v_{\beta}(-\vec{k})=0,
\end{gathered}
$$

where $\alpha, \beta= \pm$. These relations hold in view of $a_{+}^{+}(\vec{k}) a_{+}(-\vec{k})=a_{-}^{+}(\vec{k}) a_{-}(-\vec{k})=0$. In view of the relation $\left(\gamma^{\mu} k_{\mu}-\gamma^{5} m\right)^{2}=k^{2}+m^{2}=E^{2}-\vec{k}^{2}+m^{2}$, the generalization of these solutions to the massive tachyonic Dirac 
equation is rather straightforward. We find

$$
\begin{aligned}
U_{+}(\vec{k}) & =\frac{\gamma^{5} m-\gamma^{\mu} k_{\mu}}{\sqrt{2} \sqrt{(E-|\vec{k}|)^{2}+m^{2}}} u_{+}(\vec{k}) \\
& =\left(\begin{array}{l}
\frac{m-E+|\vec{k}|}{\sqrt{2} \sqrt{(E-|\vec{k}|)^{2}+m^{2}}} a_{+}(\vec{k}) \\
\frac{m+E-|\vec{k}|}{\sqrt{2} \sqrt{(E-|\vec{k}|)^{2}+m^{2}}} a_{+}(\vec{k})
\end{array}\right)
\end{aligned}
$$

for positive chirality and

$$
\begin{aligned}
U_{-}(\vec{k}) & =\frac{\gamma^{\mu} k_{\mu}-\gamma^{5} m}{\sqrt{2} \sqrt{(E-|\vec{k}|)^{2}+m^{2}}} u_{-}(\vec{k}) \\
& =\left(\begin{array}{l}
\frac{m+E-|\vec{k}|}{\sqrt{2} \sqrt{(E-|\vec{k}|)^{2}+m^{2}}} a_{-}(\vec{k}) \\
\frac{-m+E-|\vec{k}|}{\sqrt{2} \sqrt{(E-|\vec{k}|)^{2}+m^{2}}} a_{-}(\vec{k})
\end{array}\right)
\end{aligned}
$$

for negative chirality. Here, $E=\sqrt{\vec{k}^{2}-m^{2}}$ with $\vec{k}^{2}>m^{2}$. For $m \rightarrow 0$, the particle has to be on the mass shell, therefore $E \rightarrow|\vec{k}|$, and we have the tachyon spinors approaching their massless limit, i.e., $U_{+}(\vec{k}) \rightarrow u_{+}(\vec{k})$ and $U_{-}(\vec{k}) \rightarrow u_{-}(\vec{k})$. The negative-energy eigenstates are

$$
\begin{aligned}
V_{+}(\vec{k}) & =\frac{\gamma^{5} m+\gamma^{\mu} k_{\mu}}{\sqrt{2} \sqrt{(E-|\vec{k}|)^{2}+m^{2}}} u_{+}(\vec{k}) \\
& =\left(\begin{array}{l}
\frac{-m-E+|\vec{k}|}{\sqrt{2} \sqrt{(E-|\vec{k}|)^{2}+m^{2}}} a_{+}(\vec{k}) \\
\frac{-m+E-|\vec{k}|}{\sqrt{2} \sqrt{(E-|\vec{k}|)^{2}+m^{2}}} a_{+}(\vec{k})
\end{array}\right)
\end{aligned}
$$

for positive chirality (negative helicity) and

$$
\begin{aligned}
V_{-}(\vec{k}) & =\frac{-\gamma^{\mu} k_{\mu}-\gamma^{5} m}{\sqrt{2} \sqrt{(E-|\vec{k}|)^{2}+m^{2}}} u_{-}(\vec{k}) \\
& =\left(\begin{array}{l}
\frac{-m+E-|\vec{k}|}{\sqrt{2} \sqrt{(E-|\vec{k}|)^{2}+m^{2}}} a_{-}(\vec{k}) \\
\frac{m+E-|\vec{k}|}{\sqrt{2} \sqrt{(E-|\vec{k}|)^{2}+m^{2}}} a_{-}(\vec{k})
\end{array}\right)
\end{aligned}
$$

for negative chirality (positive helicity). For $m \rightarrow 0$ and $E \rightarrow|\vec{k}|$, we have $V_{+}(\vec{k}) \rightarrow v_{+}(\vec{k})$ and $V_{-}(\vec{k}) \rightarrow v_{-}(\vec{k})$. These states are normalized, i.e., $U_{+}^{+}(\vec{k}) U_{+}(\vec{k})=U_{-}^{+}(\vec{k}) U_{-}(\vec{k})=V_{+}^{+}(\vec{k}) V_{+}(\vec{k})=V_{-}^{+}(\vec{k}) V_{-}(\vec{k})=1$. The corresponding positive-energy solutions of the massive tachyonic Dirac equation are given as

$$
\begin{aligned}
& \Psi(x)=U_{ \pm}(\vec{k}) \mathrm{e}^{-\mathrm{i} k \cdot x}, \quad k=(E, \vec{k}), \quad E=\sqrt{\vec{k}^{2}-m^{2}}, \\
& \left(\mathrm{i} \gamma^{\mu} \partial_{\mu}-\gamma^{5} m\right) \Psi(x)=\left(\gamma^{\mu} k_{\mu}-\gamma^{5} m\right) \Psi(x)=0, \\
& \mathrm{i} \partial_{0} \Psi(x)=E \Psi(x) .
\end{aligned}
$$




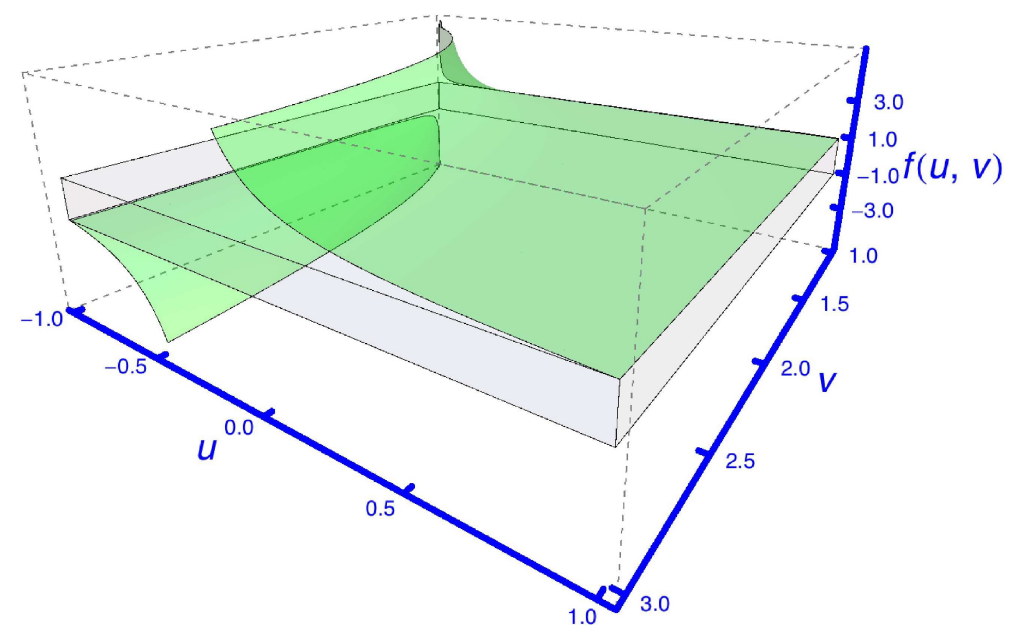

FIG. 1: (Color.) Illustration of the velocity addition theorem $f(u, v)=(u+v) /(1+u v)$, in the superluminal domain with $u \in(-1,1)$ and $v \in(1,3)$. The superluminal velocity $v$ remains superluminal upon transformation from a frame which moves at subluminal velocity $u$ with respect to the observer; the domain $-1<f(u, v)<1$ is excluded, consistent with Eq. (63).

The negative-energy solutions are given by

$$
\begin{aligned}
& \Phi(x)=V_{ \pm}(\vec{k}) \mathrm{e}^{\mathrm{i} k \cdot x}, \quad k=(E, \vec{k}), \quad E=\sqrt{\vec{k}^{2}-m^{2}}, \\
& \left(\mathrm{i} \gamma^{\mu} \partial_{\mu}-\gamma^{5} m\right) \Phi(x)=\left(-\gamma^{\mu} k_{\mu}-\gamma^{5} m\right) \Phi(x)=0, \\
& \mathrm{i} \partial_{0} \Phi(x)=-E \Phi(x),
\end{aligned}
$$

All of the discussed solutions fulfill the superluminal dispersion relation

$$
E^{2}=\vec{k}^{2}-m^{2}
$$

and are eigenstates of the tachyonic Dirac Hamiltonian (49), as given below.

\section{B. Plane-Wave Solutions and Hamiltonian}

It is instructive to investigate the Hamiltonian form of the tachyonic Dirac equation, which reads

$$
\left(\mathrm{i} \gamma^{\mu} \frac{\partial}{\partial x^{\mu}}-\gamma^{5} m\right) \psi(x)=0 \text {. }
$$

Multiplication by $\gamma^{0}$ leads to the non-covariant Hamiltonian formulation,

$$
H_{5} \psi(\vec{r})=\left(\vec{\alpha} \cdot \vec{p}+\beta \gamma^{5} m\right) \psi(\vec{r})=E \psi(\vec{r}),
$$

where $\vec{\alpha}=\gamma^{0} \vec{\gamma}$ and $\beta=\gamma^{0}$. The tachyonic Dirac Hamiltonian obviously reads as

$$
H_{5}=H_{5}(\vec{r})=\vec{\alpha} \cdot \vec{p}+\beta \gamma^{5} m,
$$

where $\vec{p}=-\mathrm{i} \vec{\nabla}$ is the momentum operator. The Hermitian adjoint is

$$
H_{5}^{+}(\vec{r})=\vec{\alpha} \cdot \vec{p}-\beta \gamma^{5} m,
$$

where by the superscript + we denote the Hermitian adjungation. Therefore,

$$
\begin{aligned}
P H_{5}^{+}(\vec{r}) P^{-1} & =P\left(\vec{\alpha} \cdot \vec{p}-\beta \gamma^{5} m\right) P^{-1} \\
& =-\vec{\alpha} \cdot \vec{p}+\beta \gamma^{5} m,
\end{aligned}
$$


because $\left(\beta \gamma^{5}\right)^{+}=\left(\gamma^{5}\right)^{+}\left(\gamma^{0}\right)^{+}=\gamma^{5} \gamma^{0}=-\gamma^{0} \gamma^{5}$. Here, $\vec{p} \rightarrow \vec{p}^{+}=\vec{p}$ because the Hermitian adjoint of the momentum operator is the momentum operator itself. It is thus easy to check that the tachyonic Hamiltonian $H_{5}$ is a pseudo-Hermitian operator [15-18, 29], with the property

$$
H_{5}(\vec{r})=P H_{5}^{+}(-\vec{r}) P^{-1}=\mathcal{P} H_{5}^{+}(\vec{r}) \mathcal{P}^{-1}
$$

where again $P=\gamma^{0}$ is the matrix representation of parity and $\mathcal{P}$ is the full parity transformation. Hamiltonians with this property have been analyzed about seventy years ago (see the work of Pauli, Ref. [29]), and have been the subject of recent investigations [10-12, 14-18] because under rather general assumptions, they have real energy eigenvalues, or resonance eigenvalues which come in complex-conjugate pairs.

This can be seen as follows and holds even though the the scalar produce $\left\langle\psi_{1}|\mathcal{P}| \psi_{2}\right\rangle$ is not positive-semidefinite. First we recall that because the spectrum of a Hermitian adjoint operator consists of the complex conjugate eigenvalues, there will be an eigenvector $\phi(\vec{r})$ with eigenvalue $E^{*}$ provided there exists an eigenvector $\psi(\vec{r})$ with eigenvalue $E$,

$$
H_{5}(\vec{r}) \psi(\vec{r})=E \psi(\vec{r}), \quad H_{5}^{+}(\vec{r}) \phi(\vec{r})=E^{*} \phi(\vec{r}) .
$$

The transformation $\vec{r} \rightarrow-\vec{r}$ and the insertion of the parity matrix $P=\gamma^{0}$ leads to

$$
\begin{aligned}
H_{5}^{+}(-\vec{r}) \phi(-\vec{r}) & =E^{*} \phi(-\vec{r}), \\
P H_{5}^{+}(-\vec{r}) P^{-1}(P \phi(-\vec{r})) & =E^{*} P \phi(-\vec{r}) .
\end{aligned}
$$

By assumption, $P H_{5}^{+}(-\vec{r}) P^{-1}=H_{5}(\vec{r})$ and thus

$$
\begin{aligned}
H_{5}(\vec{r}) P \phi(-\vec{r}) & =E^{*} P \phi(-\vec{r}), \\
H_{5}(\vec{r}) \widetilde{\psi}(\vec{r}) & =E^{*} \widetilde{\psi}(\vec{r}), \quad \widetilde{\psi}(\vec{r})=P \phi(-\vec{r}) .
\end{aligned}
$$

This implies that $\widetilde{\psi}(\vec{r})=P \phi(-\vec{r})$ is an eigenvector with eigenvalue $E^{*}$. The eigenvalues of $H_{5}$ thus come in complexconjugate pairs. Furthermore, an additional quasi-pseudo-Hermitian property can be established,

$$
H_{5}(\vec{r})=-\rho H_{5}^{+}(\vec{r}) \rho^{-1}, \quad \rho=\gamma^{0} \gamma^{5}, \quad \rho=-\rho^{-1} .
$$

Going through the above derivation again, we easily find that if $E$ and $E^{*}$ are energy eigenvalues, then $-E$ and $-E^{*}$ will also be energy eigenvalues. This configuration of eigenvalues can be characterized as a "St. Andrew's cross configuration" in the complex plane (see also Ref. [30]).

For a number of operators such as the $\mathcal{P} \mathcal{T}$ symmetric variants of odd anharmonic oscillators [13, 19, 20], the eigenvalues are all real. However, for the tachyonic Dirac Hamiltonian, we shall need the full spectrum which consists of both real as well as complex resonance eigenvalues. In order to characterize the spectrum of resonances, it is instructive to show that the resonance energies of the tachyonic Dirac Hamiltonian are real in the second-order formalism. A solution of the tachyonic equation

$$
\left(\mathrm{i} \gamma^{\mu} \frac{\partial}{\partial x^{\mu}}-\gamma^{5} m\right) \psi(x)=0, \quad \psi(x)=U \mathrm{e}^{-\mathrm{i} k \cdot x},
$$

with $k=(E, \vec{k})$, must also fulfill the Hamiltonian eigenvalue equation

$$
\left(\vec{\alpha} \cdot \vec{k}+\beta \gamma^{5} m\right) U=E U,
$$

and for $\psi(x)=V \mathrm{e}^{\mathrm{i} k \cdot x}$ we have

$$
\left(-\vec{\alpha} \cdot \vec{k}+\beta \gamma^{5} m\right) V=-E V
$$

Squaring these equations, we thus have

$$
\left(\vec{\alpha} \cdot \vec{k}+\beta \gamma^{5} m\right)^{2} U=\left(\vec{k}^{2}-m^{2}\right) U=E^{2} U
$$

and

$$
\left(-\vec{\alpha} \cdot \vec{k}+\beta \gamma^{5} m\right)^{2} V=\left(\vec{k}^{2}-m^{2}\right) V=E^{2} V
$$


We here observe that the matrix $\beta \gamma^{5}$ is a representation of the imaginary unit, in view of the identity $\left(\beta \gamma^{5}\right)^{2}=-\mathbb{1}_{4 \times 4}$. Connections to other representations of the imaginary unit within superluminal spin- $1 / 2$ Hamiltonians are discussed in A. The superluminal energy-momentum dispersion relation

$$
E^{2}=\vec{k}^{2}-m^{2}
$$

(with real $E$ ) is thus fulfilled. Resonance energies $E$ of the tachyonic Dirac Hamiltonian thus have to be either completely real (vanishing imaginary part) or completely imaginary (vanishing real part), so that $E^{2}$ is real.

\section{Need for the Inclusion of Resonances and Anti-Resonances}

One might wonder about the nature of plane-wave solutions with $|\vec{k}|<m$, which according to $E=\sqrt{\vec{k}^{2}-m^{2}}$ would have imaginary energies. Denoting by $u>1$ the velocity of the particle (expressed in units of the speed of light c), we have for tachyonic particles, according to Refs. [31-35], the relations

$$
E=\frac{m}{\sqrt{u^{2}-1}}, \quad|\vec{k}|=|\vec{p}|=\frac{m u}{\sqrt{u^{2}-1}}>m .
$$

where $u$ is the particle velocity in units of the velocity of light, $c$. Superluminal Lorentz transformations have been discussed in Refs. [31, 36] (see also Fig. 1); it is impossible to 'stop' a superluminal particle, and the 'rest frame' of a superluminal particle is that of infinite velocity $[32,37]$. We conclude that since $|\vec{k}|>m$, the problematic imaginary energies which are encountered for $|\vec{k}|<m$ are excluded by the tachyonic dispersion relation. This leads to a restriction $|\vec{k}|>m$ for the permissible values in constructing stable tachyonic wave packets ("Fourier transformations") from the fundamental plane-wave solutions. In a distant analogy to the "positive definite" functions which are the subject of Bochner's theorem (Theorem IX.9 of Ref. [38]), the wave packets of tachyonic particles have to be "positive definite" in the sense that they are naturally constructed using plane waves with $|\vec{k}|>m$ in view of the tachyonic dispersion relation. (A "positive definite" function has a positive Fourier transform, or more precisely, is characterized by a Fourier transform which constitutes a positive Borel measure.)

In Refs. [31-34], considerable effort has thus been invested into the elimination of the states with $\vec{k}^{2}<m^{2}$ from the scalar tachyonic theory, because it was realized that the resonances correspond to evanescent waves. However, we here find that these states qualify themselves as resonances of the tachyonic Dirac Hamiltonian.

We define the width $\gamma$ of a resonance of the tachyonic Dirac Hamiltonian as follows,

$$
E= \pm \sqrt{\vec{k}^{2}-m^{2}-\mathrm{i} \epsilon}=\mp \mathrm{i} \frac{\Gamma}{2}, \quad \Gamma=2 \sqrt{m^{2}-\vec{k}^{2}}, \quad|\vec{k}|<m .
$$

The wave functions describing the resonances are as follows,

$$
\begin{aligned}
& R_{+}(\vec{k})=\left(\begin{array}{l}
\frac{m+\frac{i}{2} \Gamma+|\vec{k}|}{\sqrt{2} \sqrt{\vec{k}^{2}+m^{2}+\frac{1}{4} \Gamma^{2}}} a_{+}(\vec{k}) \\
\frac{m-\frac{i}{2} \Gamma-|\vec{k}|}{\sqrt{2} \sqrt{\vec{k}^{2}+m^{2}+\frac{1}{4} \Gamma^{2}}} a_{+}(\vec{k})
\end{array}\right), \\
& R_{-}(\vec{k})=\left(\begin{array}{l}
\frac{m-\frac{i}{2} \Gamma-|\vec{k}|}{\sqrt{2} \sqrt{\vec{k}^{2}+m^{2}+\frac{1}{4} \Gamma^{2}}} a_{-}(\vec{k}) \\
\frac{-m-\frac{i}{2} \Gamma-|\vec{k}|}{\sqrt{2} \sqrt{\vec{k}^{2}+m^{2}+\frac{1}{4} \Gamma^{2}}} a_{-}(\vec{k})
\end{array}\right), \\
& E=-\frac{i}{2} \Gamma=-\frac{i}{2} \sqrt{m-\vec{k}^{2}}, \vec{k}^{2}<m^{2} .
\end{aligned}
$$


The antiresonance eigenstates are

$$
\begin{gathered}
S_{+}(\vec{k})=\left(\begin{array}{l}
\frac{-m-\frac{i}{2} \Gamma+|\vec{k}|}{\sqrt{2} \sqrt{\vec{k}^{2}+m^{2}+\frac{1}{4} \Gamma^{2}}} a_{+}(\vec{k}) \\
\frac{-m+\frac{i}{2} \Gamma-|\vec{k}|}{\sqrt{2} \sqrt{\vec{k}^{2}+m^{2}+\frac{1}{4} \Gamma^{2}}} a_{+}(\vec{k})
\end{array}\right), \\
S_{-}(\vec{k})=\left(\begin{array}{l}
\frac{-m+\frac{i}{2} \Gamma-|\vec{k}|}{\sqrt{2} \sqrt{\vec{k}^{2}+m^{2}+\frac{1}{4} \Gamma^{2}}} a_{-}(\vec{k}) \\
\frac{m+\frac{i}{2} \Gamma-|\vec{k}|}{\sqrt{2} \sqrt{\vec{k}^{2}+m^{2}+\frac{1}{4} \Gamma^{2}}} a_{-}(\vec{k})
\end{array}\right), \\
E=\frac{i}{2} \Gamma=\frac{i}{2} \sqrt{m-\vec{k}^{2}}, \quad \vec{k}^{2}<m^{2} .
\end{gathered}
$$

These states are normalized, i.e., $R_{+}^{+}(\vec{k}) R_{+}(\vec{k})=R_{-}^{+}(\vec{k}) R_{-}(\vec{k})=S_{+}^{+}(\vec{k}) S_{+}(\vec{k})=S_{-}^{+}(\vec{k}) S_{-}(\vec{k})=1$. For $|\vec{k}|=m$, we have $E \rightarrow 0$, and the eigenstates read as

$$
\begin{array}{ll}
U_{+}(\vec{k}) \rightarrow R_{+}(\vec{k}) \rightarrow\left(\begin{array}{c}
a_{+}(\vec{k}) \\
0
\end{array}\right), & |\vec{k}| \rightarrow m, \\
U_{-}(\vec{k}) \rightarrow R_{-}(\vec{k}) \rightarrow\left(\begin{array}{c}
0 \\
-a_{-}(\vec{k})
\end{array}\right), & |\vec{k}| \rightarrow m .
\end{array}
$$

The negative-energy spinors tend to the following values,

$$
\begin{array}{ll}
V_{+}(\vec{k}) \rightarrow S_{+}(\vec{k}) \rightarrow\left(\begin{array}{c}
0 \\
-a_{+}(\vec{k})
\end{array}\right), & |\vec{k}| \rightarrow m, \\
V_{-}(\vec{k}) \rightarrow S_{-}(\vec{k}) \rightarrow\left(\begin{array}{c}
-a_{-}(\vec{k}) \\
0
\end{array}\right), & |\vec{k}| \rightarrow m .
\end{array}
$$

According to Eq. (63), these spinors, which correspond to a tachyon of infinite speed, correspond to the solutions which describe a tardyon spinor at rest. This point has also been made in Ref. [37].

At time $t=0$, the above vectors are eigenstates of the Hamiltonian,

$$
\begin{array}{ll}
\psi_{1, \vec{k}}(x)=U_{ \pm}(\vec{k}) \mathrm{e}^{\mathrm{i} \vec{k} \cdot \vec{r}}, & |\vec{k}| \geq m, \\
\psi_{2, \vec{k}}(x)=V_{ \pm}(-\vec{k}) \mathrm{e}^{\mathrm{i} \vec{k} \cdot \vec{r}}, & |\vec{k}| \geq m, \\
\psi_{3, \vec{k}}(x)=R_{ \pm}(\vec{k}) \mathrm{e}^{\mathrm{i} \vec{k} \cdot \vec{r}}, & |\vec{k}|<m, \\
\psi_{4, \vec{k}}(x)=S_{ \pm}(-\vec{k}) \mathrm{e}^{\mathrm{i} \vec{k} \cdot \vec{r}}, & |\vec{k}|<m,
\end{array}
$$

with $\vec{k} \in \mathbb{R}^{3}$. These form a complete set of eigenstates of the tachyonic Dirac Hamiltonian and allow us to solve the initial value problem,

$$
\mathrm{i} \partial_{t} \psi(t, \vec{r})=H_{5} \psi(t, \vec{r}), \quad \psi(0, \vec{r})=\Psi(\vec{r}),
$$

where the initial wave-function configuration is given by $\Psi(\vec{r})$. We should indicate a little caveat, here: The $U_{ \pm}(\vec{k})$ are mutually orthogonal (positive energy), even for the same $\vec{k}$, and the $V_{ \pm}(\vec{k})$ (solutions for negative energy) have the same property [see Eq. (76c) below]. The initial value problem is thus solved on the level of the relativistic quantum theory for both positive-energy as well as negative-energy wave packets. The only remaining problem is the non-unitary character of the time evolution generated by the tachyonic Dirac Hamiltonian through the resonances, and antiresonances. Such a non-unitary time-evolution in the presence of resonances is discussed in detail in Ref. [39] 
for the case of the odd anharmonic oscillator. We should clarify that the resonance solutions $R_{ \pm}(\vec{k})$ and $S_{ \pm}(\vec{k})$ are not resonances in the sense of a virtual state in a cross section (which "decays" into a final state), but constitute complexvalued resonance energies of the Hamiltonian matrix. They describe the evanescent subluminal components of a wave packet which is genuinely superluminal. According to the i $\epsilon$ prescription from Eq. (64), resonance solutions constitute the analytic continuation of positive-energy solutions for $|\vec{k}|<m$. They are exponentially damped for propagation into the future. By contrast, anti-resonance solutions describe the continuation of negative-energy solutions for $|\vec{k}|<m$, these are damped for propagation into the past. This prescription ensures that an inconsistent exponential growth of the wave packets cannot occur [1].

Our complete set of functions (not restricted to $|\vec{k}|<m$ ) allows us to represent localized wave packets in terms of a Fourier decomposition. One example of a localized wave function in a scalar theory is

$$
\delta^{3}(\vec{r})=\int \frac{\mathrm{d}^{3} k}{(2 \pi)^{3}} \mathrm{e}^{\mathrm{i} \vec{k} \cdot \vec{r}}
$$

It has the property

$$
\int \mathrm{d}^{3} r^{\prime} \delta^{3}\left(\vec{r}^{\prime}\right) \delta^{3}\left(\vec{r}+\vec{r}^{\prime}\right)=\delta^{3}(\vec{r})
$$

Likewise the function

$$
\Psi(\vec{r})=\int_{|\vec{k}|<m} \frac{\mathrm{d}^{3} k}{(2 \pi)^{3}} R_{+}(\vec{k}) \mathrm{e}^{\mathrm{i} \vec{k} \cdot \vec{r}}+\int_{|\vec{k}| \geq m} \frac{\mathrm{d}^{3} k}{(2 \pi)^{3}} U_{+}(\vec{k}) \mathrm{e}^{\mathrm{i} \vec{k} \cdot \vec{r}}
$$

has the property

$$
\int \mathrm{d}^{3} r^{\prime} \Psi^{+}\left(\vec{r}^{\prime}\right) \Psi\left(\vec{r}+\vec{r}^{\prime}\right)=\int \frac{\mathrm{d}^{3} k}{(2 \pi)^{3}}|\widetilde{\Psi}(\vec{k})|^{2} \mathrm{e}^{\mathrm{i} \vec{k} \cdot \vec{r}}=\int \frac{\mathrm{d}^{3} k}{(2 \pi)^{3}} \mathrm{e}^{\mathrm{i} \vec{k} \cdot \vec{r}}=\delta^{3}(\vec{r}) .
$$

where $\widetilde{\Psi}(\vec{k})$ is the Fourier transform of $\Psi(\vec{r})$.

Three remarks are in order. (i) In an idealized position measurement using a detector, according to the wave function collapse postulate of quantum theory, the wave function is proportional to a Dirac- $\delta$ after the measurement. It is important to be able to localize a tachyonic particle in order to measure it. According to the Heisenberg principle, the position of quantum particle cannot be measured significantly better than its Compton wavelength, but this finding does not imply that idealized position measurements are meaningless. In our formalism (see also Ref. [1]), we prefer to retain localizability of a particle rather than prevent the emergence of the small region of non-unitary time evolution in the sector $|\vec{k}|<m$. (ii) It would be somewhat unnatural to exclude the resonances and antiresonances of the tachyonic Dirac Hamiltonian from the discussion a fortiori. Canonically, resonances and antiresonances play an important role in the spectrum of quantum Hamiltonians and should receive a physical interpretation [40]. In our case, we propose an interpretation in terms of the evanescent waves that describe tardyonic components of a genuinely tachyonic wave packet. (iii) For completeness and for general interest, we note that the Hamiltonian $H^{\prime}=\vec{\alpha} \cdot \vec{p}+\mathrm{i} \beta m$, where $\mathrm{i}$ is the imaginary unit and $m$ is a real mass term, is connected with $H_{5}$ by a unitary transformation, which is constructed in $\mathrm{A}$.

With regard to remark (i) and Refs. [31-35], we observe that full unitarity cannot be preserved anyway in a tachyonic theory at the one-loop level, as observed in Ref. [41]. Although the unitarity violation at the one-loop level could be interpreted as an argument against the consistency of tachyonic field theories, we here prefer to countenance a small unitary violation [1] due to the evanescent waves than to sacrifice the localizability of a wave packet. Likewise, in Ref. [42], it was argued that tachyonic particles cannot be described by a unitary representation of the Lorentz group. In general, we have to sacrifice either unitarity or localizability in we wish to incorporate tachyons into field theory. As observed above and explained in Ref. [1], the slight violation of unitarity is restricted to a relatively small kinematic region. Upon the inclusion of the resonances, we observe that one can write field commutators like those encountered in Eq. (1.4) of Ref. [34] in terms of the full Dirac- $\delta$ function instead of the "filtered" Dirac- $\delta$ function given in Eq. (1.5) of Ref. [34], which is not localized. This is explained in detail in Ref. [1].

With regard to remark (iii), we note that the field theory based on the Hamiltonian $H^{\prime}$ was analyzed in Ref. [37]; this analysis has recently been enhanced in Ref. [30].

\section{Orthogonality Properties of the Solutions}

Finally, a discussion of the orthogonality properties of the solutions of the tachyonic Dirac equation is in order. Let us suppose that $\psi_{2}$ is an eigenvector of a pseudo-Hermitian Hamiltonian $H_{5}$ with eigenvalues $E_{2}$ and $\psi_{1}$ is an 
eigenvector of $H_{5}$ with eigenvalues $E_{1}$. Then,

$$
\begin{aligned}
\left\langle\psi_{1}|\mathcal{P}| \psi_{2}\right\rangle & \equiv \int \mathrm{d}^{3} x \psi_{1}^{+}(\vec{x}) \gamma^{0} \psi_{2}(-\vec{x}), \\
E_{2}\left\langle\psi_{1}|\mathcal{P}| \psi_{2}\right\rangle & =\left\langle\psi_{1}\left|\mathcal{P} E_{2}\right| \psi_{2}\right\rangle=\left\langle\psi_{1}\left|\mathcal{P} H_{5}\right| \psi_{2}\right\rangle \\
& =\left\langle\psi_{1}\left|\mathcal{P} H_{5} \mathcal{P}^{-1} \mathcal{P}\right| \psi_{2}\right\rangle=\left\langle\psi_{1}\left|H_{5}^{+} \mathcal{P}\right| \psi_{2}\right\rangle \\
& =\left\langle H_{5} \psi_{1}|\mathcal{P}| \psi_{2}\right\rangle=E_{1}^{*}\left\langle\psi_{1}|\mathcal{P}| \psi_{2}\right\rangle
\end{aligned}
$$

i.e., eigenvectors for different energy are orthogonal with respect to the $\mathcal{P}$-scalar product. Our plane-wave solution form a complete set of eigenstates of the Hamiltonian $H_{5}$, with the properties

$$
\begin{aligned}
H_{5} U_{ \pm}(\vec{k}) \mathrm{e}^{\mathrm{i} \vec{k} \cdot \vec{r}} & =E U_{ \pm}(\vec{k}) \mathrm{e}^{\mathrm{i} \vec{k} \cdot \vec{r}}, \\
H_{5} V_{ \pm}(-\vec{k}) \mathrm{e}^{\mathrm{i} \vec{k} \cdot \vec{r}} & =-E V_{ \pm}(-\vec{k}) \mathrm{e}^{\mathrm{i} \vec{k} \cdot \vec{r}}, \\
U_{\alpha}^{+}(\vec{k}) U_{\beta}(\vec{k}) & =\delta_{\alpha \beta}, \quad V_{\alpha}^{+}(-\vec{k}) V_{\beta}(-\vec{k})=\delta_{\alpha \beta}, \\
U_{\alpha}^{+}(\vec{k}) \gamma^{0} V_{\beta}(+\vec{k}) & =0, \quad \alpha, \beta= \pm,
\end{aligned}
$$

and $E=\sqrt{\vec{k}^{2}-m^{2}}$. The relation $U_{\alpha}^{+}(\vec{k}) \gamma^{0} V_{\beta}(+\vec{k})=0$ is equivalent to

$$
\left\langle U_{ \pm}(\vec{k}) \mathrm{e}^{\mathrm{i} \vec{k} \cdot \vec{r}}|\mathcal{P}| V_{ \pm}(\vec{k}) \mathrm{e}^{-\mathrm{i} \vec{k} \cdot \vec{r}}\right\rangle=\left\langle U_{ \pm}(\vec{k}) \mathrm{e}^{\mathrm{i} \vec{k} \cdot \vec{r}}\left|\gamma^{0}\right| V_{ \pm}(+\vec{k}) \mathrm{e}^{\mathrm{i} \vec{k} \cdot \vec{r}}\right\rangle=0
$$

which is equivalent to the orthogonality of the eigenvectors with different energies $E$ and $-E$ under the scalar product defined in Eq. (75). Within the space of positive-energy and negative-energy solutions, the time evolution is unitary with respect to the ordinary scalar product defined in Eq. (76c). Furthermore, we observe that while in general $U_{\alpha}^{+}(\vec{k}) V_{\beta}(-\vec{k}) \neq 0$, the four solutions $U_{ \pm}(\vec{k}) \mathrm{e}^{\mathrm{i} \vec{k} \cdot \vec{r}}$ and $V_{ \pm}(-\vec{k}) \mathrm{e}^{\mathrm{i} \vec{k} \cdot \vec{r}}$ form a linearly independent set for given $\vec{k}$. However, as explained in $\mathrm{B}$, the $\mathcal{P}$ norm of the plane-wave states with a nonvanishing $\vec{k}$ actually vanishes, and the $\mathcal{P}$ scalar product therefore is not positive definite. Therefore, as explained in detail in Ref. [43], it would be desirable to work with a different scalar product, obtained by prepending the $\mathcal{P}$ operator with a second operator, called $\mathcal{C}$ in Ref. [43] (while being manifestly different from charge conjugation, see B), so that the ensuing redefined scalar product is positive-definite.

The construction of a positive-definite scalar product, conserved under the pseudo-Hermitian time evolution, is left as an open problem for future investigations. However, one important observation can be made at the current stage: namely, the time evolution induced by the pseudo-Hermitian Hamiltonian is separately unitary for wave packets constructed from exclusively positive-energy and negative-energy wave packets, in view of the property (76c) and the reality of the eigenvalues. This pseudo-unitarity is sufficient if one assumes that the Hamiltonian naturally breaks up into a positive-energy and negative-energy Hamiltonian, according to the interpretation of positive-energy solutions describing particles and negative-energy solutions describing antiparticles [1]. Indeed, for a Hermitian Hamiltonian, the scalar product

$$
\left\langle\psi_{1}(t) \mid \psi_{2}(t)\right\rangle=\int \mathrm{d}^{3} x \psi^{+}(\vec{r}, t) \psi(\vec{r}, t)
$$

is conserved, as can be seen by differentiation with respect to time. For a pseudo-Hermitian Hamiltonian which fulfills Eq. (52), time evolution is unitary with respect to the scalar product $\left\langle\psi_{1}(t)|\mathcal{P}| \psi_{2}(t)\right\rangle$ (see Ref. [29]). This is because

$$
\begin{aligned}
& \mathrm{i} \partial_{t}\left\langle\psi_{1}(t)|\mathcal{P}| \psi_{2}(t)\right\rangle=\int \mathrm{d}^{3} x\left(-\mathrm{i} \partial_{t} \psi_{1}(t, \vec{r})\right)^{+} \mathcal{P} \psi_{2}(t, \vec{r})+\int \mathrm{d}^{3} x \psi_{1}^{+}(t, \vec{r}) \mathcal{P} \mathrm{i} \partial_{t} \psi_{2}(t, \vec{r}) \\
& \quad=\int \mathrm{d}^{3} x\left(-H_{5}(\vec{r}) \psi_{1}(t, \vec{r})\right)^{+} \mathcal{P} \psi_{2}(t, \vec{r})+\int \mathrm{d}^{3} x \psi_{1}^{+}(t, \vec{r}) \mathcal{P} H_{5}(\vec{r}) \psi_{2}(t, \vec{r}) \\
& \quad=-\left\langle\psi_{1}(t)\left|H_{5}^{+}(\vec{r}) \mathcal{P}\right| \psi_{2}\right\rangle+\left\langle\psi_{1}\left|\mathcal{P} H_{5}(\vec{r})\right| \psi_{2}\right\rangle=0,
\end{aligned}
$$


where we use the property $H_{5}^{+}(\vec{r}) \mathcal{P}=\mathcal{P} H_{5}(\vec{r})$. Thus, $\left\langle\psi_{1}(t)|\mathcal{P}| \psi_{2}(t)\right\rangle$ is conserved under pseudo-Hermitian time evolution. However, for wave packets constructed from plane waves that fulfill the superluminal dispersion relation (63), we can actually show a stronger statement because the energy eigenvalues $E_{\vec{k}}=\sqrt{\vec{k}^{2}-m^{2}}$ with $|\vec{k}|>m$ are purely real. Let us study two positive-energy normalized wave packets of the form $(j=1,2)$,

$$
\begin{aligned}
& \psi_{j}(t, \vec{r})=\int \frac{\mathrm{d}^{3} k}{(2 \pi)^{3}}\left(w_{j,+}(\vec{k}) U_{+}(\vec{k})+w_{j,-}(\vec{k}) U_{-}(\vec{k})\right) \mathrm{e}^{-\mathrm{i} E_{\vec{k}} t+\mathrm{i} \vec{k} \vec{r}} \\
& \int \mathrm{d}^{3} k\left(\left|w_{j,+}(\vec{k})\right|^{2}+\left|w_{j,-}(\vec{k})\right|^{2}\right)=1
\end{aligned}
$$

where the $U_{ \pm}(\vec{k})$ are given in Eq. (42). Then,

$$
\begin{aligned}
\left\langle\psi_{1}(t, \vec{r}) \mid \psi_{2}(t, \vec{r})\right\rangle= & \int \mathrm{d}^{3} x \int \frac{\mathrm{d}^{3} k^{\prime}}{(2 \pi)^{3}} \int \frac{\mathrm{d}^{3} k^{\prime \prime}}{(2 \pi)^{3}}\left(w_{1,+}^{*}\left(\vec{k}^{\prime}\right) U_{+}^{+}\left(\vec{k}^{\prime}\right)+w_{1,-}^{*}\left(\vec{k}^{\prime}\right) U_{-}^{+}\left(\vec{k}^{\prime}\right)\right) \\
& \times\left(w_{2,+}\left(\vec{k}^{\prime \prime}\right) U_{+}\left(\vec{k}^{\prime \prime}\right)+w_{2,-}\left(\vec{k}^{\prime \prime}\right) U_{-}\left(\vec{k}^{\prime \prime}\right)\right) \mathrm{e}^{-\mathrm{i}\left(E_{\vec{k}^{\prime \prime}}-E_{\vec{k}^{\prime}}\right) t+\mathrm{i}\left(\vec{k}^{\prime \prime}-\vec{k}^{\prime}\right) \cdot \vec{r}} \\
= & \int \frac{\mathrm{d}^{3} k^{\prime}}{(2 \pi)^{3}} \int \mathrm{d}^{3} k^{\prime \prime}\left(w_{1,+}^{*}\left(\vec{k}^{\prime}\right) w_{2,+}\left(\vec{k}^{\prime \prime}\right) U_{+}^{+}\left(\vec{k}^{\prime}\right) U_{+}\left(\vec{k}^{\prime \prime}\right)\right. \\
& \left.+w_{1,-}^{*}\left(\vec{k}^{\prime}\right) w_{2,-}\left(\vec{k}^{\prime \prime}\right) U_{-}^{+}\left(\vec{k}^{\prime}\right) U_{-}\left(\vec{k}^{\prime \prime}\right)\right) \mathrm{e}^{-\mathrm{i}\left(E_{\vec{k}^{\prime \prime}}-E_{\vec{k}^{\prime}}\right) t} \delta^{3}\left(\vec{k}^{\prime \prime}-\vec{k}^{\prime}\right) \\
= & \int \frac{\mathrm{d}^{3} k}{(2 \pi)^{3}}\left(w_{1,+}^{*}(\vec{k}) w_{2,+}(\vec{k})+w_{1,-}^{*}(\vec{k}) w_{2,-}(\vec{k})\right) \\
= & \left\langle\psi_{1}(0, \vec{r}) \mid \psi_{2}(0, \vec{r})\right\rangle .
\end{aligned}
$$

Thus, unitary time evolution holds provided the energies $E_{\vec{k}}=\sqrt{\vec{k}^{2}-m^{2}}$ fulfill the dispersion relation given in Eq. (63), which implies that they are manifestly real. Within the manifolds of positive-energy and negative-energy wave packets, the $\mathcal{C}$ operator in the sense of $\mathrm{B}$ thus can be taken to be equal to the parity operator $\mathcal{P}$, in accordance with the findings of Ref. [44].

\section{CONCLUSIONS}

In the current article, the tachyonic Dirac equation $\left(\mathrm{i} \gamma^{\mu} \partial_{\mu}-\gamma^{5} m\right) \psi=0$ is analyzed. We show that it is invariant under time reversal $(\mathcal{T}$, Sec. II C), and invariant under charge conjugation and parity $(\mathcal{C P})$. Our formulation of the time reversal operation involves the Dirac adjoint (see Sec. 4.4 .2 of Ref. [25]). We generalize the treatment of the $\mathcal{C}$, $\mathcal{P}$ and $\mathcal{T}$ transformations to the tachyonic Dirac equation in Secs. II A, II B and II C. These are the desired symmetry properties for the description of neutrinos. The solutions of the tachyonic equation approximate the chiral solutions of the massless Dirac equation in the limit $m \rightarrow 0$ (see Sec. III A). In writing the solutions, one is naturally led to the use of a basis of chiral eigenfunctions, aligned with the propagation four-momentum $k=(E, \vec{k})$ of the particle. The tachyonic Hamiltonian given in Eq. (49), $H_{5}=\vec{\alpha} \cdot \vec{p}+\beta \gamma^{5} m$, is shown to be pseudo-Hermitian. The concept of pseudo-Hermiticity is related to an article of Pauli [29] who established Hamiltonians with the given property as viable alternatives to Hermitian Hamiltonians (see also Refs. [10-12, 14]). In particular, pseudo-Hermiticity has been indispensable in the recent generalization of the so-called Bender-Wu formulas [45-47] to odd anharmonic oscillators $[19,20]$.

In the tachyonic Dirac Hamiltonian $H_{5}=\vec{\alpha} \cdot \vec{p}+\beta \gamma^{5} m$, the matrix $\mathrm{i}_{4}=\beta \gamma^{5}$ is a representation of the imaginary unit in four dimensions,

$$
\mathrm{i}_{4}=\beta \gamma^{5}=\left(\begin{array}{cc}
0 & \mathbb{1}_{2 \times 2} \\
-\mathbb{1}_{2 \times 2} & 0
\end{array}\right), \quad\left(\mathrm{i}_{4}\right)^{2}=-\mathbb{1}_{4 \times 4},
$$

where $\mathbb{1}_{2 \times 2}$ denotes the $2 \times 2$ unit matrix, and $\mathbb{1}_{4 \times 4}$ denotes the $4 \times 4$ unit matrix. Indeed, it was Dirac who argued $[48,49]$ that the linearization of the Klein-Gordon equation required the introduction of $4 \times 4$ matrices; the most straightforward implementation of the imaginary unit in four-dimensional space is due to the matrix $i_{4}=\beta \gamma^{5}$ because it generalizes the two-dimensional representation of the complex imaginary unit. Indeed, it is well known that complex multiplication can be written as matrix multiplication within the group of matrices of the form

$$
z=|z|\left(\begin{array}{cc}
\cos \theta & \sin \theta \\
-\sin \theta & \cos \theta
\end{array}\right)=\left(\begin{array}{cc}
\operatorname{Re} z & \operatorname{Im} z \\
-\operatorname{Im} z & \operatorname{Re} z
\end{array}\right)
$$


where $z=|z| \exp (\mathrm{i} \theta)$ is a complex number and the imaginary unit has $|z|=1$ and $\theta=\pi / 2$. Division algebras ("matrix multiplication algebras") without zero divisors only exist in 1, 2, 4 and 8 dimensions [50, 51].

We have discussed the description of a tachyonic wave packet using a pseudo-Hermitian Hamiltonian whose planewave solutions fulfill the tachyonic dispersion relation. The Hamiltonians $H_{5}=\vec{\alpha} \cdot \vec{p}+\beta \gamma^{5} \mathrm{~m}$, or alternatively $H^{\prime}=\vec{\alpha} \cdot \vec{p}+\mathrm{i} \beta m$, have pseudo-Hermitian as well as quasi-pseudo-Hermitian properties, as discussed in Sec. III A. The description leads to a unitary time evolution within wave packets that are composed exclusively of positiveenergy and negative-energy waves. The scalar product employed in the calculation of the unitary time evolution is the ordinary scalar product, and it is positive-definite for positive-energy and negative-energy wave packets. Positiveenergy solutions are commonly interpreted as those characterizing particles, and negative-energy solution characterize antiparticles. It has recently been shown [1] that for neutrinos, the field-theoretical generalization of the derivation presented here leads to a natural suppression of right-handed neutrinos and left-handed anti-neutrinos, due to an indefinite Hilbert-space norm of the "wrong-helicity" states which is induced by helicity-dependent anticommutators, as explained in Ref. [1]. On the level of quantum mechanics, the construction of a conserved (under time evolution) scalar product applicable to both positive-energy as well as negative-energy wave packets is left as an open problem. However, we stress that due to the i $\epsilon$ prescription as employed in Ref. [1] in the tachyonic propagator denominator, positive-energy and negative-energy solutions propagate independently.

Inconclusive indications for a possibly superluminal nature of the neutrino come from low-energy beta decay experiments [52-58], from an early arrival of neutrinos from the 1987 supernova [59], from the MINOS laboratory-based time-of-flight experiment (as reported in Ref. [60]), as well as from an earlier FERMILAB experiment [61]. The OPERA experiment [62] is currently being reexamined. While none of these experiments provides conclusive evidence for the superluminal nature of the neutrino, it is interesting to observe that the experimentally determined best estimate of the neutrino propagation velocity has been measured as superluminal in Refs. [60-62], and the best estimates for the neutrino mass squares have been determined to be negative in Refs. [52-58], with a tendency toward smaller absolute values for the mass squares at lower energies (for a summary see also [63]). Conceivable energy-dependent neutrino mass running is discussed in Refs. [64, 65].

\section{Appendix A: Unitary Transformations of the Superluminal Hamiltonian}

In Refs. [30, 37], the Hamiltonian

$$
H^{\prime}=\vec{\alpha} \cdot \vec{p}+\mathrm{i} \beta m,
$$

is investigated, where $\mathrm{i}$ is the imaginary unit and $m$ is a real mass term. This Hamiltonian is related to the tachyonic Dirac Hamiltonian as follows. We consider the unitary transformation

$$
\begin{aligned}
U & =\frac{1}{\sqrt{2}}\left[\left(\frac{1}{2}+\frac{\mathrm{i}}{2}\right)\left(1-\gamma^{0} \gamma^{5}\right)+\left(\frac{1}{2}-\frac{\mathrm{i}}{2}\right)\left(\gamma^{0}+\gamma^{5}\right)\right] \gamma^{1} \\
& =\left(\begin{array}{cccc}
0 & \frac{\mathrm{i}}{\sqrt{2}} & 0 & \frac{1}{\sqrt{2}} \\
\frac{\mathrm{i}}{\sqrt{2}} & 0 & \frac{1}{\sqrt{2}} & 0 \\
0 & -\frac{\mathrm{i}}{\sqrt{2}} & 0 & \frac{1}{\sqrt{2}} \\
-\frac{\mathrm{i}}{\sqrt{2}} & 0 & \frac{1}{\sqrt{2}} & 0
\end{array}\right)
\end{aligned}
$$

which has the property

$$
U \beta \gamma^{5} U^{-1}=\mathrm{i} \beta, \quad \widetilde{\alpha}^{i}=U \alpha^{i} U^{-1}, \quad\left\{\alpha^{i}, \alpha^{j}\right\}=\left\{\widetilde{\alpha}^{i}, \widetilde{\alpha}^{j}\right\}=2 \delta^{i j} .
$$

Now,

$$
H^{\prime \prime}=U H_{5} U^{-1}=U\left(\alpha^{i} p^{i}+\beta \gamma^{5} m\right) U^{-1}=\widetilde{\alpha}^{i} p^{i}+\mathrm{i} \beta m .
$$

Because the $\widetilde{\alpha}^{i}$ fulfill the same algebraic relations as the $\alpha^{i}$, the Hamiltonian $H^{\prime \prime}$ is equivalent to the Hamiltonian $\left(\widetilde{\alpha}^{i} \rightarrow \alpha^{i}\right)$

$$
H^{\prime}=\alpha^{i} p^{i}+\mathrm{i} \beta m .
$$

Using a further unitary transformation, we may invert the sign of the imaginary mass term,

$$
H^{\prime \prime \prime}=\gamma^{5} H^{\prime}\left(\gamma^{5}\right)^{-1}=\alpha^{i} p^{i}-\mathrm{i} \beta m,
$$

where $\beta^{-1}=\beta$ is a unitary operator. Because of the unitary relation of $H^{\prime \prime \prime}, H^{\prime \prime}, H^{\prime}$, and $H_{5}$, it is not surprising that the field theories induced by the Hamiltonians are indeed equivalent, as analyzed in detail in Ref. [30] (with a special emphasis on $H_{5}$ and $H^{\prime}$ ). 


\section{Appendix B: Quantum Mechanics and Scalar Products}

We attempt to investigate the connection to quantum mechanical Hamiltonians as described in Refs. [10-14]. For definiteness, we follow the conventions of Ref. [43], which implies that the operators $\mathcal{P}$ and $\mathcal{T}$ are restricted to the domain of ordinary (nonrelativistic) quantum mechanical operators, and from now one, $\mathcal{C}$ is redefined to be a pseudo charge-conjugation in accordance with Ref. [43]. Hamiltonians of the following structure are being investigated,

$$
H=p^{2}+x^{2}(\mathrm{i} x)^{\epsilon}=-\frac{\partial^{2}}{\partial x^{2}}+x^{2}(\mathrm{i} x)^{\epsilon},
$$

where $p=-\mathrm{i} \partial / \partial x$ is the momentum operator. In Refs. [10-14, 43], the parity operator is defined to be equivalent to the transformation $x \rightarrow-x, p \rightarrow-p$, while the $\mathcal{T}$ operator describes the time inversion, which is equivalent to $x \rightarrow x$, $p \rightarrow-p$, and $\mathrm{i} \rightarrow-\mathrm{i}$. We note that the transformation $\mathrm{i} \rightarrow-\mathrm{i}$ is equivalent to the calculation of the Hermitian adjoint. For the class of Hamiltonians given by Eq. (B1), which we consider exclusively from now on, the $\mathcal{T}$ transformation is equivalent to the calculation of the Hermitian adjoint, and we have

$$
[\mathcal{P} \mathcal{T}, H]=0, \quad H=\mathcal{P} \mathcal{T} H \mathcal{T}^{-1} \mathcal{P}^{-1}=\mathcal{P} H^{+} \mathcal{P}^{-1} .
$$

In the case of unbroken $\mathcal{P} \mathcal{T}$ symmetry, the eigenfunctions of the Hamiltonian are also eigenfunctions of the $\mathcal{P} \mathcal{T}$ operator [43],

$$
H \phi_{n}(x)=E_{n} \phi_{n}(x), \quad \mathcal{P} \mathcal{T} \phi_{n}(x)=\phi_{n}(x) .
$$

Using the Hermiticity of the $\mathcal{P}$ operator, we can formulate the condition

$$
(f, g)=\int \mathrm{d} x[\mathcal{P} \mathcal{T} f(x)] g(x)=\int \mathrm{d} x \mathcal{T} f(x) \mathcal{P} g(x)=\int \mathrm{d} x f^{*}(x) \mathcal{P} g(x),
$$

which is equivalent to our $\mathcal{P}$ scalar product discussed in Sec. III D. Now, for the case of a discrete spectrum, one uses Eq. (B3) and obtains [43]

$$
\left(\phi_{n}, \phi_{m}\right)=\int \mathrm{d} x \phi_{n}^{*}(-x) \phi_{m}(x)=\int \mathrm{d} x \phi_{n}(x) \phi_{m}(x)=(-1)^{n} \delta_{n m} .
$$

A scalar product is positive definite if its matrix representation is positive definite in a complete basis set of functions. Let us choose the basis set to be the set of the eigenstates $\phi_{n}(x)$. Then, if we can find an operator $\mathcal{C}$ which has the following properties,

$$
\mathcal{C} \phi_{n}(x)=(-1)^{n} \phi_{n}(x), \quad\langle f \mid g\rangle \equiv \int \mathrm{d} x[\mathcal{C P} \mathcal{T} f(x)] g(x), \quad\left\langle\phi_{n} \mid \phi_{m}\right\rangle=\delta_{n m},
$$

the scalar product $\langle\cdot \mid \cdot\rangle$ is positive definite. This defines the $\mathcal{C}$ operator whose calculation is discussed in Refs. [43, 44].

The above formalism is somewhat tied to systems with a discrete spectrum of bound states. For the continuum eigenstates of the tachyonic Dirac Hamiltonian, the norm in the $\mathcal{P}$ scalar product vanishes because for plane-wave states of wave vector $\vec{k}$, it is proportional to the expression

$$
\begin{aligned}
\int \mathrm{d}^{3} x[\exp (\mathrm{i} \vec{k} \cdot \vec{x})]^{*} \mathcal{P} \exp (\mathrm{i} \vec{k} \cdot \vec{x}) & =\int \mathrm{d}^{3} x \exp (-\mathrm{i} \vec{k} \cdot \vec{x}) \exp (-\mathrm{i} \vec{k} \cdot \vec{x}) \\
& =(2 \pi)^{3} \delta^{3}(2 \vec{k})=0, \quad \vec{k} \neq \overrightarrow{0} .
\end{aligned}
$$

The physically interesting states have a nonvanishing wave vector and thus, zero $\mathcal{P}$ norm. A possible choice for the $\mathcal{C}$ operator, which is physically consistent within the manifold of positive-energy and negative-energy states, therefore is $\mathcal{C}=\mathcal{P}$, as discussed in Sec. III D. By "physically consistent" we mean that it leads to a conserved scalar product within the time evolution for the positive- and negative-energy states.

\section{Acknowledgments}

This work was supported by the NSF and by the National Institute of Standards and Technology (precision measurement grant).

[1] U. D. Jentschura and B. J. Wundt, Eur. Phys. J. C 72, 1894 (2012). 
[2] C. R. Galley, B. L. Hu, and S.-Y. Lin, Phys. Rev. D 74, 024017 (2006).

[3] P. J. Mohr, Ann. Phys. (N.Y.) 325, 607 (2010).

[4] A. Chodos, A. I. Hauser, and V. A. Kostelecky, Phys. Lett. B 150, 431 (1985).

[5] A. Chodos, V. A. Kostelecky, R. Potting, and E. Gates, Mod. Phys. Lett. A 7, 467 (1992).

[6] A. Chodos and V. A. Kostelecky, Phys. Lett. B 336, 295 (1994).

[7] T. Chang, A new Dirac-type equation for tachyonic neutrinos, e-print arXiv:hep-th/0011087.

[8] T. Chang, Nucl. Sci. Technol. 13, 129 (2002).

[9] V. K. Oikonomou, The 2d Gross-Neveu Model for Pseudovector Fermions and Tachyonic Mass Generation, e-print arXiv:1109.6170.

[10] C. M. Bender and S. Boettcher, Phys. Rev. Lett. 80, 5243 (1998).

[11] C. M. Bender and G. V. Dunne, J. Math. Phys. 40, 4616 (1999).

[12] C. M. Bender, S. Boettcher, and P. N. Meisinger, J. Math. Phys. 40, 2201 (1999).

[13] C. M. Bender and E. J. Weniger, J. Math. Phys. 42, 2167 (2001).

[14] C. M. Bender, D. C. Brody, and H. F. Jones, Phys. Rev. Lett. 89, 270401 (2002).

[15] A. Mostafazadeh, J. Math. Phys. 43, 205 (2002).

[16] A. Mostafazadeh, J. Math. Phys. 43, 2814 (2002).

[17] A. Mostafazadeh, J. Math. Phys. 43, 3944 (2002).

[18] A. Mostafazadeh, J. Math. Phys. 44, 974 (2003).

[19] U. D. Jentschura, A. Surzhykov, and J. Zinn-Justin, Phys. Rev. Lett. 102, 011601 (2009).

[20] U. D. Jentschura, A. Surzhykov, and J. Zinn-Justin, Ann. Phys. (N.Y.) 325, 1135 (2010).

[21] C. M. Bender, H. F. Jones, and R. J. Rivers, Phys. Lett. B 625, 333 (2005).

[22] K. Jones-Smith and H. Mathur, A New Class of non-Hermitian Quantum Hamiltonians with PT symmetry, e-print arXiv:0908.4255.

[23] K. Jones-Smith and H. Mathur, Relativistic Non-Hermitian Quantum Mechanics, e-print arXiv:0908.4257.

[24] C. Itzykson and J. B. Zuber, Quantum Field Theory (McGraw-Hill, New York, 1980).

[25] H. van Hees, Introduction to Relativistic Quantum Field Theory, Lecture Scriptum, available at http://fias.unifrankfurt.de/ ${ }^{\sim}$ hees/publ/lect.pdf.

[26] M. E. Peskin and D. V. Schroeder, An Introduction to Quantum Field Theory (Perseus, Cambridge, Massachusetts, 1995).

[27] I. Białynicki-Birula and Z. Białynicka-Birula, Quantum Electrodynamics (Pergamon Press, Oxford, 1975).

[28] M. Srednicki, Quantum Field Theory (Cambridge University Press, Cambridge, 2007).

[29] W. Pauli, Rev. Mod. Phys. 15, 175 (1943).

[30] U. D. Jentschura, Dirac Equation with Imaginary Mass and Helicity-Dependence, e-print arXiv:1201.6300, submitted (2012).

[31] O. M. P. Bilaniuk, V. K. Deshpande, and E. C. G. Sudarshan, Am. J. Phys. 30, 718 (1962).

[32] G. Feinberg, Phys. Rev. 159, 1089 (1967).

[33] M. E. Arons and E. C. G. Saudarshan, Phys. Rev. 173, 1622 (1968).

[34] J. Dhar and E. C. G. Saudarshan, Phys. Rev. 174, 1808 (1968).

[35] O.-M. Bilaniuk and E. C. G. Sudarshan, Nature (London) 223, 386 (1969).

[36] R. I. Sutherland and J. R. Shepanski, Phys. Rev. D 33, 2896 (1986).

[37] J. Bandukwala and D. Shay, Phys. Rev. D 9, 889 (1974).

[38] M. Reed and B. Simon, Methods of Modern Mathematical Physics, Volume II: Fourier Analysis, Self-Adjointness (Academic Press, San Diego, California, 1978).

[39] U. D. Jentschura, A. Surzhykov, M. Lubasch, and J. Zinn-Justin, J. Phys. A 41, 095302 (2008).

[40] M. Reed and B. Simon, Methods of Modern Mathematical Physics, Volume III: Scattering Theory (Academic Press, San Diego, California, 1978).

[41] D. G. Boulware, Phys. Rev. D 1, 2426 (1970).

[42] L. Xinzhou and L. Jizong, J. Phys. A 20, 6113 (1987).

[43] C. M. Bender, J. Brod, A. Refig, and M. E. Reuter, J. Phys. A 37, 10139 (2004).

[44] C. M. Bender, S. F. Brandt, J. H. Chen, and Q. Wang, Phys. Rev. D 71, 065010 (2005).

[45] C. M. Bender and T. T. Wu, Phys. Rev. 184, 1231 (1969).

[46] C. M. Bender and T. T. Wu, Phys. Rev. Lett. 27, 461 (1971).

[47] C. M. Bender and T. T. Wu, Phys. Rev. D 7, 1620 (1973).

[48] P. A. M. Dirac, Proc. Roy. Soc. London A 117, 610 (1928).

[49] P. A. M. Dirac, Proc. Roy. Soc. London A 118, 351 (1928).

[50] R. Bott and J. Milnor, Bull. Amer. Math. Soc. 64, 87 (1958).

[51] M. Kervaire, Proc. Natl. Acad. Sci. USA 44, 280 (1958).

[52] R. G. H. Robertson, T. J. Bowles, G. J. Stephenson, D. L. Wark, J. F. Wilkerson, and D. A. Knapp, Phys. Rev. Lett. 67, 957 (1991).

[53] K. Assamagan, C. Brönnimann, M. Daum, H. Forrer, R. Frosch, P. Gheno, R. Horisberger, M. Janousch, P.-R. Kettle, T. Spirig, and C. Wigger, Phys. Lett. B 335, 231 (1994).

[54] W. Stoeffl and D. J. Decman, Phys. Rev. Lett. 75, 3237 (1995).

[55] K. Assamagan, C. Brönnimann, M. Daum, H. Forrer, R. Frosch, P. Gheno, R. Horisberger, M. Janousch, P. R. Kettle, T. Spirig, and C. Wigger, Phys. Rev. D 53, 6065 (1996).

[56] C. Weinheimer, B. Degen, A. Bleile, J. Bonn, L. Bornschein, O. Kazachenko, A. Kovalik, and E. Otten, Phys. Lett. B 460, 
219 (1999).

[57] V. M. Lobashev, V. N. Aseev, A. I. Belesev, A. I. Berlev, E. V. Geraskin, A. A. Golubev, O. V. Kazachenko, Y. E. Kuznetsov, R. P. Ostroumov, L. A. Ryvkis, B. E. Stern, N. A. Titov, S. V. Zadorozhny, and Y. I. Zakharov, Phys. Lett. B 460, 227 (1999).

[58] A. I. Belesev, E. V. Geraskin, B. L. Zhuikov, S. V. Zadorozhny, O. V. Kazachenko, V. M. Kohanuk, N. A. Lihovid, V. M. Lobasheva, A. A. Nozik, V. I. Parfenov, A. K. Skasyrskaya, E. A. Sudachkov, N. A. Titiov, and V. G. Usanov, Phys. At. Nucl. 71, 449 (2008).

[59] V. L. Dadykin et al., JETP 45, 593 (1987).

[60] P. Adamson et al., Phys. Rev. D 76, 072005 (2007).

[61] G. R. Kalbfleisch, N. Baggett, E. C. Fowler, and J. Alspector, Phys. Rev. Lett. 43, 1361 (1979).

[62] M. Antonello et al., ICARUS Collaboration, Measurement of the neutrino velocity with the ICARUS detector at the CNGS beam, e-print arXiv:1203.3433v1.

[63] see the URL http://cupp.oulu.fi/neutrino/nd-mass.html.

[64] M. Li and T. Wang, Mass-dependent Lorentz Violation and Neutrino Velocity, arXiv:1109.5924.

[65] U. D. Jentschura, Tachyonic Field Theory and Neutrino Mass Running, Central Eur. J. Phys. (2012), in press. 